${ }^{1}$ Centro Cardiovascular, Hospital Clínico Regional de Concepción Dr. Guillermo Grant Benavente. Concepción, Chile. 2Departamento de Cirugía, Facultad de Medicina, Universidad de Concepción. Concepción, Chile.

${ }^{3}$ Servicio de Cirugía, Hospital Clínico Regional de Concepción Dr. Guillermo Grant Benavente. Concepción, Chile. ${ }^{4}$ Unidad de Anatomía Patológica, Hospital Clínico Regional de Concepción Dr. Guillermo Grant Benavente. Concepción, Chile. aEstudiante Medicina, Facultad de Medicina, Universidad de Concepción. Concepción, Chile.

Los autores declaran no tener conflictos de interés. Trabajo no recibió financiamiento.

Recibido el 21 de enero de 2020, aceptado el 16 de junio de 2020 .

Correspondencia a: Roberto González Lagos Departamento de Cirugía, Facultad de Medicina, Universidad de Concepción, Janequeo esquina Chacabuco S/N. Concepción, Chile. rgonzalezlagos@udec.cl

\section{Hidatidosis pulmonar: características, manifestaciones clínicas y tratamiento en pacientes hospitalizados en Concepción, Región del Biobío, Chile}

\author{
ROBERTO GONZÁLEZ L. ${ }^{1,2}$, ALEJANDRA RIQUELME U. ${ }^{3}$, \\ RODRIGO REYES M. ${ }^{1,2}$, FELIPE ALARCÓN O. ${ }^{a}$, \\ LORETO SPENCER L. ${ }^{4}$, SEBASTIÁN BARRA M. ${ }^{a}$, \\ ENRIQUE SEGUEL S. ${ }^{1,2}$, ALECK STOCKINS L. ${ }^{1,2}$, \\ ANDRÉS JADUE T. ${ }^{1}$, DIEGO SALDIVIA Z. ${ }^{a}$, \\ ANDRÉS SCHAUB C. ${ }^{a}$, EMILIO ALARCÓN C. ${ }^{1,2}$
}

\section{Features of pulmonary hydatidosis in 368 patients admitted to a regional hospital}

Background: Lungs are the second location in frequency of hydatidosis or cystic echinococcosis. Aim: To characterize the clinical manifestations, diagnosis, findings and treatment of patients hospitalized for a pulmonary hydatid cyst (PHC). Materials and Methods: Review of databases, medical records, operation notes and death certificates of patients admitted to a Chilean regional hospital with a PHC. Results: Four hundred twenty-nine cases were identified and complete data was obtained from 368 patients aged $34 \pm 19$ years, 224 (60,9\%) men. The most common clinical manifestations were cough in $269(73 \%)$ and chest pain in 217 (59\%). The most frequent locations were the right lung in 210 (57\%) and lower lobes in 218 (59\%). One hundred eighty-seven cysts (51\%) were complicated. Conservative surgery (cystectomy) was performed in 308 (84\%). Postoperative morbidity was observed in $77(21 \%)$ and mortality in $6(2 \%)$ patients. Recurrence was observed in 28 (8\%) patients. There was a significant reduction in morbidity, mortality, reoperations, and postoperative days over time. Conclusions: In these patients, pulmonary hydatidosis was diagnosed mainly during working ages and half of patients had a complicated cyst.

(Rev Med Chile 2020; 148: 762-771)

Key words: Echinococcosis, Pulmonary; Lung Diseases; Parasitic Diseases; Thoracic Surgery; Zoonoses.
L a hidatidosis o echinococcosis quística es una zoonosis parasitaria de distribución mundial, siendo la especie Echinococcus granulosus a la cual se atribuye la totalidad de los casos publicados en Chile ${ }^{1,2}$.

En Sudamérica, el hospedero definitivo de importancia epidemiológica es el perro y los animales de abasto (principalmente ovino y caprino) son los hospederos intermediarios que completan el ciclo del parásito. La transmisión al humano ocurre cuando este ingiere los huevos eliminados en las heces del huésped definitivo ${ }^{3,4}$.

La hidatidosis humana es la principal causa de hospitalización por enfermedad parasitaria en Chile, tiene alta morbilidad y costos socioeconómicos que incluyen pérdidas por días de ausencia laboral, hospitalización, tratamientos médicos, exámenes y procedimientos quirúrgicos ${ }^{5}$. 
En nuestro país, la hidatidosis es una enfermedad de notificación obligatoria (ENO) con una incidencia aproximada de 2-2,2 casos por cada 100.000 habitantes, sin embargo, existe una importante subnotificación que se evidencia por la disparidad entre los datos ENO y los egresos hospitalarios por hidatidosis ${ }^{6,7}$.

La prevalencia de esta enfermedad sigue siendo desconocida, entre otras razones, por el extenso período asintomático que la caracteriza. Estudios de tamizaje mediante radiografía de tórax portátil en zonas de alta endemia en Latinoamérica reportaron prevalencias de hidatidosis de $2 \%$, estimándose que los resultados de estudios hospitalarios pueden ser 100 veces menores que la prevalencia encontrada en los estudios de tamizaje ${ }^{8}$.

En Chile, la Región de Aysén posee la mayor tasa de incidencia con 38,2 casos por 100.000 habitantes, pero es la Región del Bio Bío la que concentra el mayor número absoluto de casos a nivel país ${ }^{6}$, alcanzando aproximadamente 70 notificaciones cada año, según datos $\mathrm{ENO}$, siendo nuestro hospital el que concentra la mayor experiencia de la región en la patología.

Los pulmones son la segunda localización en frecuencia de la hidatidosis, después del hígado, representando entre $20 \%$ y $40 \%$ de los casos. El parásito alcanza los pulmones principalmente sorteando el filtro hepático vía circulación portal, sin embargo, también es posible la diseminación al romperse un quiste primario ${ }^{9,10}$.

Nuestros objetivos son caracterizar las manifestaciones clínicas, diagnóstico, hallazgos y tratamiento de los pacientes hospitalizados por quiste hidatídico pulmonar (QHP).

\section{Material y Método}

Estudio analítico de pacientes hospitalizados por QHP en Hospital Clínico Regional "Dr. Guillermo Grant Benavente" de Concepción, Chile, entre enero de 1973 y diciembre de 2018. De un total de 429 episodios registrados en el período, se obtuvieron datos completos de 368 de ellos.

El estudio se realizó con imagenología (radiografía de tórax y tomografía computada) y exámenes serológicos (enzimoinmunoanálisis de adsorción para inmunoglobulina $\mathrm{E}$ y para inmunoglobulina $\mathrm{G}$, inmunoelectroforesis para arco 5 de Capron y hemoaglutinación indirecta) dependiendo de disponibilidad de los estudios durante el período.

Se describen edad, sexo, características clínicas, estudio diagnóstico, tratamiento, estadía hospitalaria, morbilidad, mortalidad postoperatoria y recidiva.

Se consideró QHP complicado aquel que presentó evidencia clínica, imagenológica o intraoperatoria de: infección, rotura hacia árbol bronquial, rotura hacia la cavidad pleural o membrana retenida. En un episodio de QHP que involucró más de un quiste (QHP múltiple) pudieron coexistir quistes complicados y no complicados, en este caso se consideró al episodio como un QHP complicado.

$\mathrm{Al}$ describir la lateralidad del QHP, en el caso de que un episodio tenga una recidiva, si los quistes tuvieron lateralidad diferente, se registraron ambas. En el caso de la ubicación, si un paciente presentó quistes en dos o más lóbulos se consideró cada ubicación por separado.

La información fue recolectada a partir de protocolos de QHP realizados durante la estadía hospitalaria, revisión de base de datos del equipo de Cirugía Cardiotorácica, protocolos quirúrgicos, fichas clínicas, registros de anatomía patológica y certificados de defunción del Registro Civil e Identificación de Chile.

Se ordenaron los datos mediante planilla de Microsoft Excel ${ }^{\circledR}$ y se realizó análisis estadístico con el programa $S P S S 25^{\circledR}$, con función chi cuadrado para las variables cualitativas y con las pruebas t-Student y ANOVA para las variables cuantitativas. Se consideró significativo $\mathrm{p}<0,05$.

El estudio y análisis de las hospitalizaciones por hidatidosis torácica fue aprobado por el comité ético-científico de nuestra institución.

\section{Resultados}

Durante el período se hospitalizaron 429 pacientes, de estos, 269 (62,7\%) eran hombres. La edad promedio fue de 34,0 \pm 19,4 años. Se registró una mortalidad a 30 días, según información de Registro Civil, en 6 (1,4\%) pacientes (Tabla 1 ).

De un total de 368 pacientes con datos completos, $224(60,9 \%)$ eran hombres. La edad promedio fue de 34,4 \pm 19,0 años (Tabla 2).

Las manifestaciones clínicas más frecuentes fueron: tos en $269(73,1 \%)$, dolor torácico en 217 
Tabla 1. Caracterización del total de pacientes con quiste hidatídico pulmonar en el período

\begin{tabular}{|c|c|}
\hline Total de episodios QHP & 429 \\
\hline $\begin{array}{c}\text { Edad (años) } \\
\text { Promedio } \\
\text { Mediana }\end{array}$ & $\begin{array}{l}34,0 \pm 19,4 \\
31\end{array}$ \\
\hline $\begin{array}{l}\text { Sexo } \\
\text { Hombre } \\
\text { Mujer }\end{array}$ & $\begin{array}{l}269(62,7 \%) \\
160(37,3 \%)\end{array}$ \\
\hline $\begin{array}{l}\text { Características del QHP } \\
\text { Quiste no complicado } \\
\text { Quiste complicado }\end{array}$ & $\begin{array}{l}210(49,0 \%) \\
219(51,0 \%)\end{array}$ \\
\hline Mortalidad a 30 días & $6(1,4 \%)$ \\
\hline
\end{tabular}

QHP: Quiste hidatídico pulmonar.

$(59,0 \%)$ y disnea en $150(40,8 \%)$ pacientes. Fueron asintomáticos $29(7,9 \%)$ y su diagnóstico fue con imagen por otro motivo. El estudio imagenológico mostró una radiografía de tórax sospechosa de QHP en $312(84,8 \%)$ y se realizó tomografía computada de tórax (disponible desde enero de 1991) en 155 (42,1\%) casos. Los síntomas, signos y el estudio serológico se detallan en la Tabla 3.

La mayoría de los QHP se localizaron en el pulmón derecho en $210(57,1 \%)$ y en los lóbulos inferiores en $218(59,2 \%)$ casos, siendo el lóbulo inferior derecho la ubicación más frecuente. Se registró coexistencia con hidatidosis extrapulmonar en 87 (23,6\%) episodios. La localización de los quistes se detalla en la Tabla 4.

En cuanto a las características de los quistes, $79(21,5 \%)$ casos fueron múltiples, el tamaño promedio fue $9,1 \pm 4,9 \mathrm{~cm}$ y en su mayoría fueron uniloculares. En relación con el estado del quiste, se obtuvo una distribución similar entre QHP complicados y no complicados. Las complicaciones más frecuentes fueron la infección del quiste en $97(26,4 \%)$ casos y la rotura hacia la vía aérea en $82(22,3 \%)$ (Tabla 5$)$.

El tratamiento fue quirúrgico en 357 (97,0\%) casos (Figuras 1 y 2). Los 11 episodios restantes no se operaron, principalmente por rechazo del paciente a la cirugía o por enfermedad múltiple sin indicación quirúrgica. En estos pacientes se indicó como único tratamiento farmacoterapia con benzimidazoles. La cirugía fue conservadora (quistectomía) en 308 (83,7\%) episodios, dentro de este grupo se encuentran cirugías con y sin
Tabla 2. Caracterización de los pacientes con quiste hidatídico pulmonar con datos completos en el período

\begin{tabular}{|ll|}
\hline Total de episodios QHP & 368 \\
\hline $\begin{array}{l}\text { Edad (en años) } \\
\text { Promedio }\end{array}$ & $34,4 \pm 19,0$ \\
Mediana & 32 \\
$\begin{array}{l}\text { Sexo } \\
\text { Hombre }\end{array}$ \\
$\begin{array}{l}\text { Mujer } \\
\text { Domicilio rural al momento del } \\
\text { diagnóstico }\end{array}$ & $224(60,9 \%)$ \\
\hline
\end{tabular}

QHP: Quiste hidatídico pulmonar.

Tabla 3. Síntomas, signos y estudio diagnóstico del quiste hidatídico pulmonar

\begin{tabular}{|lr|}
\hline Total de episodios QHP & 368 \\
\hline Síntomas y signos & \\
Tos & $269(73,1 \%)$ \\
Dolor torácico & $217(59,0 \%)$ \\
Disnea & $150(40,8 \%)$ \\
Hemoptisis & $138(37,5 \%)$ \\
Vómica hidatídica & $99(26,9 \%)$ \\
Fiebre & $87(23,6 \%)$ \\
Asintomáticos & $29(7,9 \%)$ \\
Radiografías tórax sospechosas de QHP & $312(84,8 \%)$ \\
Opacidad redondeada & $219(70,2 \%)$ \\
\hline TC tórax realizado & $155(42,1 \%)$ \\
Eosinofilia & $168(45,7 \%)$ \\
Serología realizada & $118(32,1 \%)$ \\
Serología positiva & $84(71,2 \%)$ \\
\hline
\end{tabular}

QHP: Quiste hidatídico pulmonar; TC: Tomografía computada.

capitonaje. Se realizó cirugía radical (anatómica) en $39(10,6 \%)$ episodios, este subgrupo comprendió segmentectomías atípicas, lobectomías y neumonectomías. En 33 (9,0\%) casos, asociado o no a cirugía conservadora o radical, se realizaron cirugías relacionadas con la complicación del quiste; como decorticación pleuropulmonar, reparación diafragmática, cierre de fístulas, entre otras. La morbilidad, mortalidad, reoperaciones, estadía postoperatoria y recidiva se detallan en Tabla 6. 
Tabla 4. Lateralidad, ubicación y quistes asociados al quiste hidatídico pulmonar

\begin{tabular}{|lr|}
\hline Total de episodios QHP & 368 \\
Lateralidad & \\
Derecho & $210(57,1 \%)$ \\
Izquierdo & $153(41,6 \%)$ \\
Bilateral & $35(9,5 \%)$ \\
Lóbulos superiores & $154(41,8 \%)$ \\
Izquierdo & $86(23,4 \%)$ \\
Derecho & $68(18,5 \%)$ \\
Lóbulo medio & $37(10,1 \%)$ \\
Lóbulos inferiores & $218(59,2 \%)$ \\
Izquierdo & $86(23,4 \%)$ \\
Derecho & $132(35,9 \%)$ \\
Tránsito hepato-torácico & $10(2,7 \%)$ \\
Quistes en otra localización & $87(23,6 \%)$ \\
Hepático & $74(20,1 \%)$ \\
Peritoneal & $9(2,4 \%)$ \\
Renal & $3(0,8 \%)$ \\
Esplénico & $3(0,8 \%)$ \\
Pared torácica & $3(0,8 \%)$ \\
Pericárdico & $2(0,5 \%)$ \\
Músculo deltoides & $2(0,5 \%)$ \\
Mediastino & $1(0,3 \%)$ \\
\hline
\end{tabular}

QHP: Quiste hidatídico pulmonar.

En el seguimiento, se realizó cirugía tardía por morbilidad de secuela parenquimatosa pulmonar postquirúrgica (principalmente bronquiectasias sintomáticas y aspergiloma en cavidades residuales) en $10(2,8 \%)$ casos, realizándose mayoritariamente lobectomías, con un promedio de 82 meses desde la cirugía primaria.

Al comparar los resultados según el estado del quiste se observó mortalidad, morbilidad, necesidad de reoperación y estadía hospitalaria significativamente mayores en los QHP complicados versus los no complicados. En cuanto a la cirugía conservadora versus radical, solo se observó diferencia significativa en mortalidad. Al comparar las técnicas específicas se observaron diferencias entre los distintos grupos (Tablas 7 y 8 ).

En los resultados quirúrgicos según períodos se observó una disminución significativa de la mortalidad, morbilidad, necesidad de reoperación y estadía hospitalaria (Tabla 9).
Tabla 5. Características y estado del quiste hidatídico pulmonar

\begin{tabular}{|lc|}
\hline Total de episodios QHP & 368 \\
\hline Características del quiste & \\
Único & $289(78,5 \%)$ \\
Múltiple & $79(21,5 \%)$ \\
Quiste unilocunar & $335(91,0 \%)$ \\
Tamaño promedio (cm) & $9,1 \pm 4,9$ \\
Estado del QHP & \\
Quiste no complicado & $187(50,8 \%)$ \\
Quiste complicado & $181(49,2 \%)$ \\
Infección & $97(26,4 \%)$ \\
Rotura hacia la vía aérea & $82(22,3 \%)$ \\
Membrana retenida & $58(15,8 \%)$ \\
Rotura hacia la pleura & $28(7,6 \%)$ \\
\hline
\end{tabular}

QHP: Quiste hidatídico pulmonar.

\section{Tabla 6. Tratamiento, resultados del tratamiento quirúrgico y recidiva del quiste hidatídico pulmonar}

\begin{tabular}{|lc|}
\hline Total de episodios QHP & 368 \\
\hline Tratamiento quirúrgico & $357(97,0 \%)$ \\
\hline Conservadora & $308(83,7 \%)$ \\
$\quad$ Quistectomía con capitonaje & $209(56,8 \%)$ \\
Quistectomía sin capitonaje & $99(26,9 \%)$ \\
\hline Radical & $39(10,6 \%)$ \\
$\quad$ Lobectomía & $24(6,5 \%)$ \\
Segmentectomía atípica & $12(3,3 \%)$ \\
Neumonectomía & $3(0,8 \%)$ \\
Otra cirugía & $33(9,0 \%)$ \\
\hline Tratamiento farmacológico asociado & $91(24,7 \%)$ \\
Morbilidad & $77(20,9 \%)$ \\
$\quad$ Fistula broncopleural & $38(10,3 \%)$ \\
Empiema & $11(3,0 \%)$ \\
Hemotórax & $8(2,2 \%)$ \\
Otros & $25(6,8 \%)$ \\
\hline Mortalidad & $6(1,6 \%)$ \\
\hline Reoperaciones & $30(8,2 \%)$ \\
\hline Estadía postoperatoria (días) & \\
Promedio & $13,2 \pm 11,6$ \\
Mediana & 10 \\
\hline Seguimiento & \\
$\quad$ Promedio (años) & $24,8 \pm 13,4$ \\
Recidiva & $(2,8 \%)$ \\
\hline Cirugía tardía* & \\
\hline
\end{tabular}

QHP: Quiste hidatídico pulmonar; *Principalmente lobectomías por morbilidad de secuela parenquimatosa pulmonar postquirúrgica. Las causas más frecuentes fueron bronquiectasias sintomáticas y aspergiloma en cavidades residuales. 


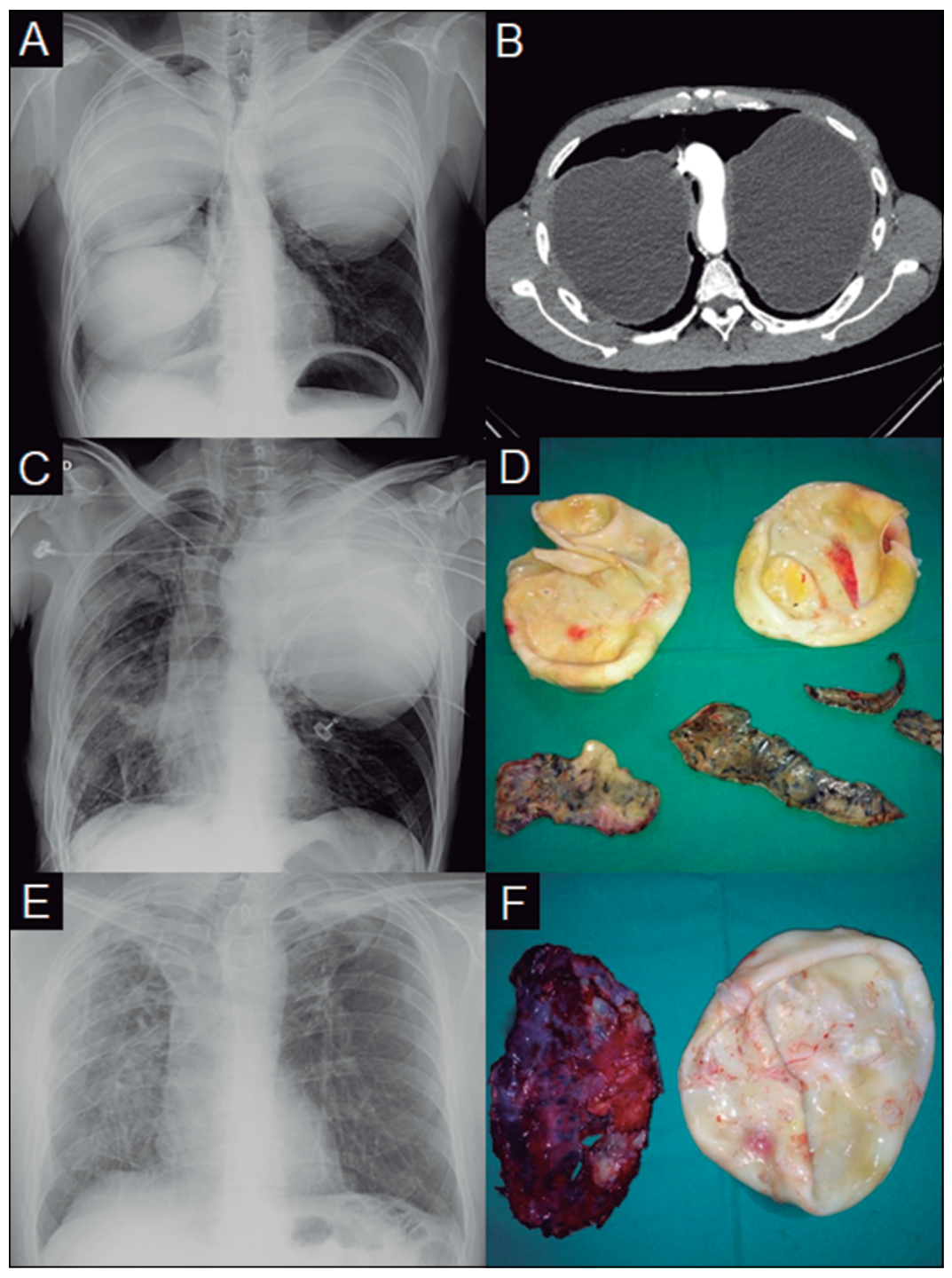

Figura 1. Hombre de 53 años que consultó por estridor y disnea, el estudio imagenológico evidenció quistes bilaterales con compresión y desviación traqueal. El grupo familiar de este hombre está compuesto por ocho individuos cuya actividad económica es la ganadería ovina, a partir de este caso se diagnosticó hidatidosis en siete de ellos. A y B: Radiografía y tomografía computada de tórax al ingreso; C y D: Radiografía de tórax postoperatoria del primer tiempo quirúrgico y macroscopía de quistes hidatídicos pulmonares derechos resecados; E y F: Radiografía de tórax postoperatoria del segundo tiempo quirúrgico y macroscopía de quiste hidatídico pulmonar izquierdo resecado.

Tabla 7. Morbilidad, mortalidad, reoperaciones y estadía postoperatoria según estado del quiste hidatídico pulmonar y tipo de técnica quirúrgica

\begin{tabular}{|c|c|c|c|c|c|c|}
\hline & $\begin{array}{c}\text { QHP } \\
\text { no complicado } \\
(187)\end{array}$ & $\begin{array}{c}\text { QHP } \\
\text { complicado } \\
(181)\end{array}$ & $\mathbf{p}$ & $\begin{array}{c}\text { Cirugía } \\
\text { conservadora } \\
(308)\end{array}$ & $\begin{array}{l}\text { Cirugía } \\
\text { radical } \\
(39)\end{array}$ & $\mathbf{p}$ \\
\hline Mortalidad & $0 \quad(0 \%)$ & $6 \quad(3,3 \%)$ & $0,012^{*}$ & $3 \quad(1,0 \%)$ & $2 \quad(5,1 \%)$ & 0,040 * \\
\hline Morbilidad & $30(16,0 \%)$ & $47(26,0 \%)$ & $0,019 *$ & $64(20,8 \%)$ & $8(20,5 \%)$ & 0,969 \\
\hline Reoperaciones & $8 \quad(4,3 \%)$ & $22(12,2 \%)$ & $0,006^{*}$ & $24 \quad(7,8 \%)$ & $5(12,8 \%)$ & 0,285 \\
\hline $\begin{array}{l}\text { Postoperatorio** } \\
\text { Promedio } \\
\text { Mediana }\end{array}$ & $\begin{array}{c}10,6 \pm 7,7 \\
8\end{array}$ & $\begin{array}{c}16,0 \pm 14,2 \\
11\end{array}$ & $<0,001 *$ & $\begin{array}{c}13,0 \pm 11,0 \\
10\end{array}$ & $\begin{array}{c}12,3 \pm 10,6 \\
10\end{array}$ & 0,739 \\
\hline
\end{tabular}

QHP: Quiste hidatídico pulmonar; *Estadísticamente significativo; **Estadía en días. 


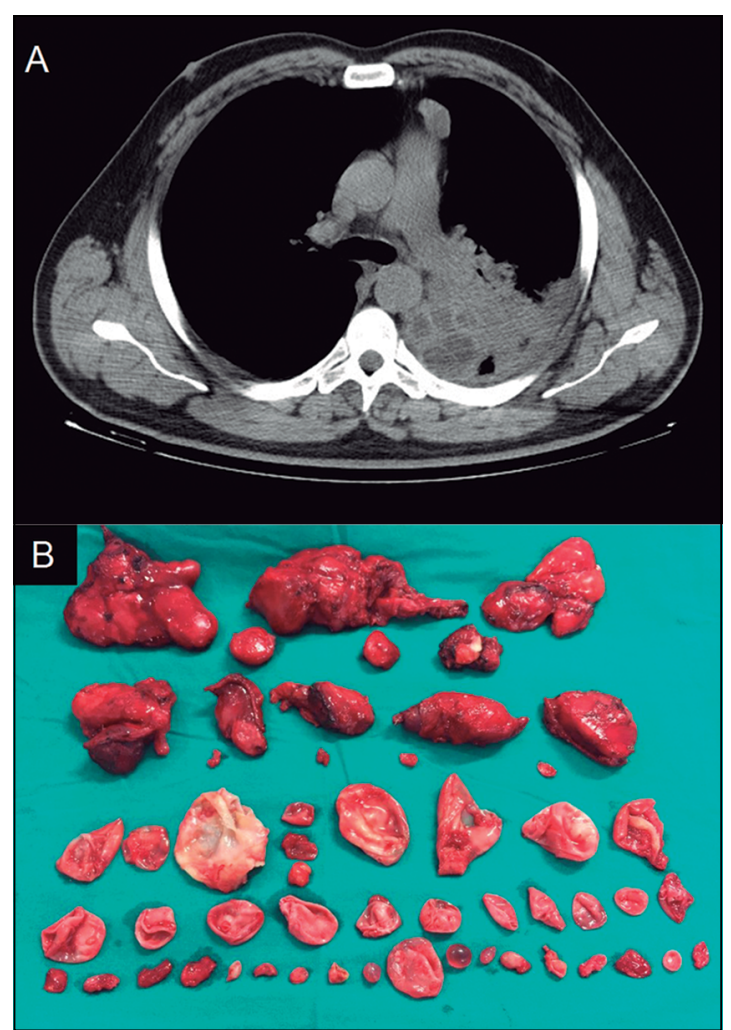

Figura 2. Hombre de 47 años que consultó por dolor torácico. A: Tomografía computada, se observan imágenes quísticas agrupadas en racimos, compatible con hidatidosis pulmonar complicada con implantación secundaria pleural; B: Macroscopía de parénquima pulmonar (con quistes) y quistes resecados

\section{Discusión}

La hidatidosis continúa siendo una enfermedad con alto impacto socioeconómico a nivel nacional y mundial ${ }^{1,5-7}$. En nuestra serie, la hidatidosis pulmonar se presentó en adultos en plena edad productiva y cerca de la mitad de los casos presentó alguna complicación al momento del diagnóstico. En relación a los resultados inmediatos del tratamiento quirúrgico, la morbilidad, mortalidad, reoperaciones y estadía postoperatoria han disminuido significativamente a través del tiempo.

El diagnóstico precoz de la hidatidosis se dificulta debido a su largo período asintomático. En el pulmón, el diámetro del quiste aumenta a una velocidad estimada de un centímetro por año, dependiendo de la elasticidad del tejido pulmonar adyacente, la reserva pulmonar y la respuesta inmune del paciente ${ }^{11}$. En nuestra serie, el diámetro promedio proyecta un período de aproximadamente una década desde la infección del parásito hasta el diagnóstico.

La compliance pulmonar permite el crecimiento del QHP de forma asintomática hasta que el tamaño provoca una compresión de tejidos circundantes con la consecuente aparición de tos y dolor torácico. Por su parte, el QHP complicado con rotura hacia los bronquios puede presentar expectoración hemoptoica, hemoptisis o vómica hidatídica, y al romperse hacia la pleura se mani-

Tabla 8. Morbilidad, mortalidad, reoperaciones y estadía postoperatoria según tipo de técnica quirúrgica

\begin{tabular}{|c|c|c|c|c|c|}
\hline & \multicolumn{2}{|c|}{ Cirugía conservadora (308) } & \multicolumn{3}{|c|}{ Cirugía radical (39) } \\
\hline & $\begin{array}{c}\text { Quistectomía } \\
\text { capitonaje } \\
\text { (209) }\end{array}$ & $\begin{array}{c}\text { Quistectomía } \\
\text { sin capitonaje } \\
\text { (99) }\end{array}$ & $\begin{array}{l}\text { Lobectomía } \\
\text { (24) }\end{array}$ & $\begin{array}{c}\text { Segmentectomía } \\
\text { (12) }\end{array}$ & $\begin{array}{c}\text { Neumonectomía } \\
\text { (3) }\end{array}$ \\
\hline Mortalidad & $2(1,0 \%)$ & $1 \quad(1,0 \%)$ & $1 \quad(4,2 \%)$ & $0(0,0 \%)$ & $1(33,3 \%)$ \\
\hline Morbilidad & $44(21,1 \%)$ & $20(20,2 \%)$ & $6(25,0 \%)$ & $0(0,0 \%)$ & $2(66,7 \%)$ \\
\hline Reoperaciones & $15 \quad(7,2 \%)$ & $9 \quad(9,1 \%)$ & $3(12,5 \%)$ & $0(0,0 \%)$ & $2(66,7 \%)$ \\
\hline \multicolumn{6}{|l|}{ Postoperatorio** } \\
\hline Promedio & $12,3 \pm 9,2$ & $14,3 \pm 13,9$ & $11,8 \pm 5,6$ & $9,3 \pm 9,3$ & $28,7 \pm 28,9$ \\
\hline Mediana & 10 & 8 & 10 & 6 & 13 \\
\hline
\end{tabular}

**Estadía en días. 
Tabla 9. Morbilidad, mortalidad, reoperaciones y estadía postoperatoria según período

\begin{tabular}{|lcccc|}
\hline & $\mathbf{1 9 7 3 - 1 9 8 9 ( 1 3 8 )}$ & $\mathbf{1 9 9 0 - 2 0 0 9 ( 1 6 8 )}$ & $\mathbf{2 0 1 0 - 2 0 1 8 ~ ( 6 2 )}$ & p \\
\hline Mortalidad & $3(2,2 \%)$ & $3(1,8 \%)$ & $0(0,0 \%)$ & $0,047^{*}$ \\
Morbilidad & $34(24,6 \%)$ & $37(22,0 \%)$ & $6(9,7 \%)$ & $0,049 *$ \\
$\begin{array}{l}\text { Reoperaciones } \\
\begin{array}{l}\text { Postoperatorio** } \\
\text { Promedio }\end{array}\end{array}$ & $13(9,4 \%)$ & $15(8,9 \%)$ & $2(3,2 \%)$ & $0,029 *$ \\
Mediana & $16,4 \pm 11,9$ & $12,0 \pm 11,7$ & $9,6 \pm 9,0$ & $<0,001^{*}$ \\
\hline
\end{tabular}

*Estadísticamente significativo; **Estadía en días.

festará con clínica de ocupación pleural y posible posterior empiema ${ }^{10}$.

Budke y cols. ${ }^{8}$, en un metaanálisis sobre las manifestaciones clínicas del QHP reportaron frecuencias de $51,3 \%$ de tos, $39,9 \%$ de dolor torácico y $8,8 \%$ de vómica hidatídica; valores comparativamente menores a los encontrados en nuestra serie, donde la vómica hidatídica alcanzó $26,9 \%$. Las manifestaciones alérgicas (que pueden presentarse desde un exantema leve hasta la anafilaxia) son poco consignadas y generalmente subdiagnosticadas.

El diagnóstico de QHP se plantea ante una imagen compatible asociada al antecedente de la exposición en zonas endémicas. El estudio se realiza mediante imágenes y serología en la mayoría de los casos ${ }^{9}$.

En nuestra serie se observó una radiografía sospechosa de QHP en $84,8 \%$ de los episodios y en su mayoría se observó la imagen clásica de QHP correspondiente a una opacidad redondeada, homogénea y bien delimitada. El patrón radiográfico cambia posterior a la complicación del quiste, siendo la imagen más frecuente la rotura contenida, observada como membranas flotando dentro del quiste ${ }^{12}$. La tomografía computada es útil ante la duda diagnóstica, en la planificación de la cirugía, en episodios múltiples o con diseminación secundaria, entre otros, siendo actualmente un examen de rutina. El estudio de QHP debe incluir una imagen abdominal debido a la alta coexistencia de quistes hidatídicos hepáticos, que en nuestra serie alcanza a 20,1\%, sin embargo, se debe tener en cuenta que los quistes pueden localizarse en otros órganos como riñón, bazo, corazón, entre otros ${ }^{13-17}$.

El estudio serológico actual en Chile consiste en métodos de enzimoinmunoanálisis (ELISA) para cribado y Western blot como técnica de con- firmación ${ }^{13}$. En nuestra serie, se utilizaron diferentes pruebas según la disponibilidad en nuestro centro y se registró una positividad de 71,2\% de los casos. Cabe destacar que un resultado negativo no descarta el diagnóstico debido a que la positividad depende de la ubicación, viabilidad del parásito e integridad del quiste, siendo más probable que el resultado sea positivo en el QHP complicado ${ }^{18}$. Por otro lado, la sensibilidad del estudio serológico es menor en el QHP, con valores entre 50\% y 60\% en comparación con el quiste hidatídico hepático o multiorgánico, en donde tiene una sensibilidad entre $85 \%$ y $98 \%$, respectivamente ${ }^{19}$.

El QHP se describe más frecuentemente en el pulmón derecho y en lóbulos inferiores ${ }^{20}$, lo que es concordante con nuestros hallazgos. Esto se explicaría porque la principal vía de diseminación es hematógena y los lóbulos inferiores son los más perfundidos.

Cerca de la mitad de los episodios fueron QHP complicados, proporción similar a otras series ${ }^{21,22}$. La rotura del quiste es la principal causa de complicación de un QHP, este puede romperse hacia la vía aérea o hacia la cavidad pleural, en ambos casos el quiste puede infectarse, siendo esta última la complicación más frecuente en nuestra serie, de forma menos frecuente puede producir una membrana retenida. El QHP complicado se asocia a mayor daño del parénquima pulmonar, necesidad de cirugía radical, morbilidad y mortalidad que los no complicados ${ }^{18,21,22}$.

El tratamiento del QHP es quirúrgico y tiene como objetivos resecar la hidátide, evitar la rotura y diseminación intraoperatoria, cerrar las comunicaciones hacia la vía aérea, cavidad pleural o ambas, y evitar cavidades residuales. Se debe resecar la menor cantidad de parénquima pulmonar posible, realizando una cirugía radical si el parénquima adyacente está gravemente dañado 
o infectado, o cuando las áreas atelectásicas se consideran irrecuperables ${ }^{11,22-24}$. Estudios han reportado una necesidad de cirugía radical cercanas a $10 \%$, variando según el estado del quiste ${ }^{25,26}$. En nuestra serie, se realizó cirugía radical en 10,6\% de los casos.

El capitonaje se realizó en aproximadamente la mitad de los casos. Técnicamente consiste en aproximar las paredes de la cavidad residual con suturas reabsorbibles ${ }^{27}$ suturando desde profundo a superficial, lo que potencialmente reduce el riesgo de fístulas broncopleurales persistentes, infección de la cavidad residual y empiema, sin embargo, estudios han reportado resultados controversiales al compararla con la técnica sin capitonaje, no existiendo un beneficio claro ${ }^{28-31}$.

En nuestra serie se observó morbilidad en 77 $(20,9 \%)$ casos, siendo la fístula broncopleural la más frecuente. Estudios han reportado tasas de complicaciones entre 6,9\% y $39 \%$ en el postoperatorio, observándose una mayor tasa en los QHP complicados ${ }^{22,32-36}$.

Nabi y Waseem ${ }^{37}$, en una revisión sobre los resultados quirúrgicos, observaron una mortalidad perioperatoria entre $0 \%$ y $2 \%$, tanto para la cirugía conservadora como la radical, siendo similar a nuestros resultados.

Cabe destacar que, a pesar de que los principios de la técnica quirúrgica no han cambiado sustancialmente, se observó una mejora en los resultados inmediatos del tratamiento quirúrgico a través del tiempo. Esto podría deberse a mejoras en el acceso a salud, lo que permitiría un diagnóstico más precoz, optimización de la técnica quirúrgica y mejora en los cuidados intrahospitalarios.

La recidiva fue de 7,6\%, esto puede estar subestimado, ya que los pacientes en su mayoría son de zonas aisladas, donde la consulta es tardía, y debido al extenso período asintomático. Además, la recidiva se favorece por ser una población cautiva que sigue estando expuesta a los factores de riesgo.

Existen complicaciones tardías asociadas a las secuelas parenquimatosas de la cirugía del quiste hidatídico pulmonar que, en nuestra serie, requirieron reintervención en $2,8 \%$ de los casos. Sin embargo, este número no necesariamente representa al total de pacientes que presentan secuelas sintomáticas posterior a la cirugía.

El diagnóstico tardío, la dificultad de acceso a tratamiento y de seguimiento han sido asociados a una mayor mortalidad por hidatidosis en nuestro país, existiendo una asociación entre mayores tasas de mortalidad y zonas dedicadas a la economía básica y con menor acceso a las prestaciones de salud $^{38}$.

Debido a estas problemáticas, guías internacionales recomiendan que, sumado a un diagnóstico y tratamiento oportuno, se deben incluir a distintos actores que pueden contribuir a evitar la continuidad del ciclo, mediante acciones como la desparasitación de perros, vigilancia epidemiológica y el desarrollo de estudios de tamizaje en zonas de alta endemia que permitan visualizar la problemática y formular programas locales eficaces para el control y prevención de la hidatidosis ${ }^{1,39,40}$. Cabe destacar que en Chile se implementó recientemente la vacunación a hospederos intermediarios con la vacuna recombinante EG95 en zonas de alta endemia. Esta vacuna ha mostrado niveles de protección entre $82 \%$ y $99 \%$ con dos dosis ${ }^{40,41}$ y potencialmente puede generar un impacto positivo como medida de control de la enfermedad.

En conclusión, en nuestro medio, la hidatidosis pulmonar ocurre principalmente en población en edad laboralmente activa y se diagnostica tardíamente, observándose una importante proporción de QHP complicados. El tratamiento previo a la complicación del quiste es un factor que disminuye la morbilidad y mortalidad, por lo que el antecedente de exposición en zonas endémicas y la sospecha clínica son fundamentales para un diagnóstico precoz y así mejorar los resultados del tratamiento. Por último, los resultados inmediatos del tratamiento quirúrgico del quiste hidatídico pulmonar han presentado una mejoría significativa a través del tiempo.

\section{Referencias}

1. Thompson RC y McManus DP. Aetiology: parasites and life-cycles. En: Eckert J, Gemmell MA, Meslin FX, Pawlowski ZS, Editores. WHO/OIE: Manual on Echinococcosis in Humans and Animals: a Public Health Problem of Global Concern. Paris, Francia: World Organisation for Animal Health.; 2001. p.1-17.

2. Serra I. Hidatidosis alveolar, una enfermedad inexistente en Chile. Rev Chil Cir 2005; 57: 278.

3. González H, Plaza J, Abalos P. Fertilidad del quiste hidatídico en tres especies animales de Chile y estudio de la vitalidad de sus escólices. Bol Chile Parasit 1981; 36: 14-9. 
4. Rodríguez JC. Hidatidosis pulmonar. En: Rodríguez JC, Undurraga A, Editores. Enfermedades respiratorias 2nd ed. Santiago, Chile: Editorial Mediterráneo.; 2011. p. 923-38.

5. Venegas J, Espinoza S, Sánchez G. Impacto socioeconómico de la hidatidosis en Chile. Rev Med Chile 2014; 142: 1023-33.

6. Cortés S, Valle C. Hidatidosis humana: Generalidades y situación epidemiológica en Chile según egresos hospitalarios y notificación obligatoria entre los años 2001 y 2005. Rev Chilena Infectol 2010; 27: 329-35.

7. MINSAL. Subsecretaría de Salud Pública, Ministerio de Salud. Manual para el diagnóstico, tratamiento, prevención y control de la hidatidosis en Chile. [Internet]. 2015. Disponible en: http://www.repositoriodigital.minsal.cl/bitstream/handle/2015/836/Manual-Hidatidosis. pdf? sequence $=1$ \&isAllowed $=\mathrm{y}$

8. Budke C, Carabin H, Ndimubanzi P, Nguyen H, Rainwater E, Dickey $\mathrm{M}$, et al. A systematic review of the literature on cystic echinococcosis frequency worldwide and its associated clinical manifestations. Am J Trop Med Hyg 2013; 88: 1011-27.

9. Macpherson CN, Vuitton DA, Gharbi HA, Caremani M, Frider B, Brunetti E, et al. International classification of ultrasound images in cystic echinococcosis for application in clinical field epidemiological settings. Acta Trop 2003; 85: 253-61.

10. Morar R, Feldman C. Pulmonary echinococcosis. Eur Respir J 2003; 21: 1069-77.

11. Halezeroglu S, Celik M, Uysal A, Senol C, Keles M, Arman B. Giant hydatid cyst of the lung. J Thorac Cardiovasc Surg 1997; 113: 712-7.

12. Balikian J, Mudarris F. Hydatid disease of the lung. A roentgenologic study of 50 cases. Am J Roentgenol Radium Ther Nucl Med 1974; 122: 692-707.

13. Pinto P. Diagnóstico, tratamiento y seguimiento de la hidatidosis. Rev Chil Cir 2017; 69: 94-8.

14. Álvarez R, Alarcón E, González R. Quiste hidatídico retroperitoneal. Rev Chil Cir 2002: 54: 536-8.

15. González R, Reyes R, Riquelme A, Seguel E, Stockins A, Jadue A, et al. Hidatidosis cardíaca: tratamiento quirúrgico para recidiva de quiste hidatídico en ventrículo izquierdo. Rev Cir 2019; 71: 266-9.

16. Zamorano M, Sauré A, Valenzuela D, Cermenati B, Olivares P, Carreño T. Quiste esplénico primario. Rev Chil Cir 2016; 68: 19-20.

17. Abascal JM, Esquena S, Martos R, Ramírez C, Salvador C, Celma A, et al. Quiste hidatídico renal simulando hipernefroma. Actas Urol Esp 2005; 29: 223-5.

18. González R, Riquelme A, Ávalos M, Reyes R, Seguel E, Stockins A, et al. Hidatidosis pulmonar: Hallazgos y tratamiento quirúrgico en quistes complicados versus no complicados. Rev Cir 2020; 72: 311-8.

19. Barnes T, Deplazes P, Gottstein B, Jenkins D, Mathis A, Siles-Lucas M, et al. Challenges for diagnosis and control of cystic hydatid disease. Acta Trop 2012; 123 : 1-7.

20. Bagheri R, Haghi S, Amini M, Fattahi A, Noorshafiee S. Pulmonary hydatid cyst: Analysis of 1024 cases. Gen Thorac Cardiovasc Surg 2011; 59: 105-9.

21. Baquerizo A, Muñoz W, Barrientos F. Hidatidosis pulmonar: experiencia en 1094 quistes. Rev Chil Cir 1998; 50: 502-8.

22. Kuzuku A, Soysal O, Ozgel M, Yologlu S. Complicated hydatid cyst of the lung: clinical and therapeutic issues. Ann Thorac Surg 2004; 77: 1200-4.

23. Dakak M, Caylak H, Kavakli K, Gozubuyuk A, Yucel O, Gurkok S, et al. Parenchyma-saving surgical treatment of giant pulmonary hydatid cyst. J Thorac Cardiovasc Surg 2009; 57: 165-8.

24. Kavukcu S, Kilic D, Tokat AO, Kutlay H, Cangir AK, Enon S, et al. Parenchyma-preserving surgery in the management of pulmonary hydatid cyst. J Invest Surg 2006; 19: 61-8.

25. Vasquez JC, Montesinos E, Peralta J, Rojas L, De la Rosa J, Leon JJ. Need for lung resection in patients with intact or ruptured hydatid cyst. Thorac Cardiovasc Surg 2009; 57: 295-302.

26. Halezeroglu S, Okur E, Tanyu M. Surgical management for hydatid disease. Thorac Surg Clin 2012; 22: 375-85.

27. Lichter I. Surgery of pulmonary hydatid cyst-the Barrett technique. Thorax 1972; 27: 529-34.

28. Erdogan A, Ayten A, Demircan A. Methods of surgical therapy in pulmonary hydatid disease: is capitonnage advantageous? Anz J Surg 2005; 75: 992-6.

29. Kosar A, Orki A, Haciibrahimoglu G, Kiral H, Arman B. Effect of capitonnage and cystotomy on outcome of childhood pulmonary hydatid cyst. J Thorac Cardiovasc Surg 2006; 132: 560-4.

30. Bilgin M, Oguzkaya F, Akcali Y. Is capitonnage unnecessary in the surgery of intact pulmonary hydatid cyst? Anz J Surg 2004; 74: 40-2.

31. Turna A, Yilmaz M, Haciigrahimoglu G, Kutlu C, Bedirhan M. Surgical treatment of pulmonary hydatid cysts: is capitonnage necessary? Ann Thorac Surg 2002; 74: 191-5.

32. Jerray M, Benzarti M, Garrouche A, Klabi N, Hayouni A. Hydatid disease of the lungs. Study of 386 cases. Am Rev Respir Dis 1992; 146: 185-9.

33. Goshal A, Sarkal S, Saha K, Sarkar U, Kundu S, Chatterjee $S$, et al. Hydatid lung disease: an analysis of five years 
cumulative data from Kolkata. J Assoc Physicians India 2012; 60: 12-6.

34. Safioleas M, Misiakos E, Dosios T, Manti C, Lambrou P, Skalkeas G. Surgical treatment for lung hydatid disease. World J Surg 1999; 23: 1181-5.

35. Aribas O, Kanat F, Gormus N, Turk E. Pleural complications of hydatid disease. J Thorac Cardiovasc Surg 2002; 123: 492-7.

36. Fatimi S, Naureen S, Moizuddin S, Puri M, Yousuf M, Javed $M$, et al. Pulmonary hydatidosis: clinical profile and follow up from an endemic region. ANZ J Surg 2007; 77: 749-51.

37. Nabi MS, Waseem T. Pulmonary hydatid disease: What is the optimal surgical strategy? Int J Surg 2010; 8: 612-6.

38. Martínez P. Caracterización de la mortalidad por hidatidosis humana. Chile, 2000-2010. Rev Chilena Infectol 2014; 31: 7-15.
39. Acosta-Jammett G, Vargas R, Ernst S. Caracterización epidemiológica de hidatidosis humana y animal en la Región de Los Ríos, 1999-2009. Rev Chilena Infectol 2016; 33: 419-27.

40. Larrieu E, Arezo M, Pacheco de Caldas E, Casas N, Del Grande L, Del Río V, et al. Prevención y control de la hidatidosis en el nivel local: iniciativa sudamericana para el control y vigilancia de la equinococosis quística/hidatidosis. Río de Janeiro, Brasil: Editorial: Organización Panamericana de la Salud - OPS/OMS.; 2017.56 .

41. Servicio Agrícola y ganadero: SAG [Internet]. Chile; 2016. Autoridades inician programa de vacunación de ovinos para control de hidatidosis 2016 [cited 2020 abril 1]. Available from: https://www.sag.gob.cl/noticias/ autoridades-inician-programa-de-vacunacion-de-ovinos-para-control-de-hidatidosis. 